Supplement of Nat. Hazards Earth Syst. Sci., 14, 2289-2312, 2014

http://www.nat-hazards-earth-syst-sci.net/14/2289/2014/

doi:10.5194/nhess-14-2289-2014-supplement

(C) Author(s) 2014. CC Attribution 3.0 License.

(c) (i)
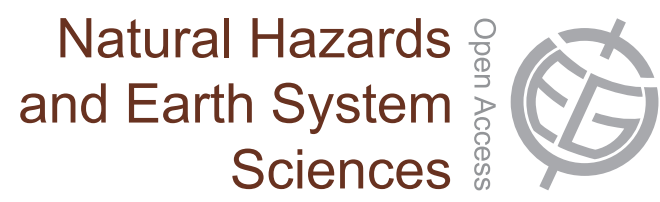

Supplement of

\title{
A multi-scale risk assessment for tephra fallout and airborne concentration from multiple Icelandic volcanoes - Part 2: Vulnerability and impact
}

\section{Scaini et al.}

Correspondence to: C. Scaini (saetachiara@gmail.com) and S. Biass (sebastien.biasse@unige.ch) 
This supplementary material comprises exposure and vulnerability maps and tables produced to compile the tephra impact assessment presented in the article "A multi-scale risk assessment for tephra fallout and airborne concentration from multiple Icelandic volcanoes - Part II: vulnerability and impact" by C. Scaini et al.

Supplementary material is constituted by:

1) Table S1. Icelandic municipalities map and identification number

2) Table S2. Vulnerable population for municipalities

3) Fig. S1. Thematic maps of milk (a) and wool (b) production and percentage of agricultural area (c) for municipalities

4) Table S3. List of airports considered for the vulnerability assessment of European airspace

5) Fig. S2. Thematic maps of air traffic and multi-modal accessibility values for European Nuts-2 regions: passengers (a) and freight (b), population (c) and multi-modal accessibility (d).

6) Fig. S3. Impact maps for FIRS produced at FL150 (top) and 300 (bottom) in case of occurrence of the considered eruptive scenarios: Hekla-2000 (a), Hekla-1947 (b), Katla-1918 (c) and Askja-1875 (d). 
1) Table S1. Icelandic municipalities map and identification number

\begin{tabular}{|c|c|}
\hline Name & ID \\
\hline Borgarfjararhreppur & 7509 \\
\hline Breidalshreppur & 7613 \\
\hline Bahreppur & 7611 \\
\hline Djúpavogshreppur & 7617 \\
\hline Fskrsfjararhr. & 7610 \\
\hline Fjarðabyggð & 7300 \\
\hline Fljtsdalshreppur & 7505 \\
\hline Mjafjararhreppur & 7605 \\
\hline Seyðisfjörður & 7000 \\
\hline Stvarhreppur & 7612 \\
\hline Sveitarflagi Hornafjrur & 7708 \\
\hline Vopnafjarðarhreppur & 7502 \\
\hline Tjörneshreppur & 6611 \\
\hline Pingeyjarsveit & 6612 \\
\hline Svalbarðsstrandarhreppur & 6601 \\
\hline Grýtubakkahreppur & 6602 \\
\hline Skútustaðahreppur & 6607 \\
\hline Aðaldælahreppur & 6609 \\
\hline Akureyri & 6000 \\
\hline Arnarneshreppur & 6506 \\
\hline Dalvíkurbyggð & 6400 \\
\hline Eyjafjarðarsveit & 6513 \\
\hline Grmseyjarhreppur & 6501 \\
\hline Grtubakkahreppur & 6602 \\
\hline Hrseyjarhreppur & 6504 \\
\hline Arnarneshreppur & 6506 \\
\hline Norðurping & 6100 \\
\hline Sktustaahreppur & 6607 \\
\hline Svalbarshreppur & 6706 \\
\hline Tjrneshreppur & 6611 \\
\hline Bessastaahreppur & 1603 \\
\hline Garabr & 1300 \\
\hline Hafnarfjrur & 1400 \\
\hline Kjsarhreppur & 1606 \\
\hline Kpavogur & 1000 \\
\hline Mosfellsbær & 1604 \\
\hline Reykjavk & 0000 \\
\hline Akrahreppur & 5706 \\
\hline Blönduós & 5604 \\
\hline Húnavatnshreppur & 5612 \\
\hline Skagabyggð & 5611 \\
\hline Hfahreppur & 5609 \\
\hline Húnaping vestra & 5508 \\
\hline
\end{tabular}




\begin{tabular}{|c|c|}
\hline Sveitarflagi Skagafjörður & 5200 \\
\hline Ásahreppur & 8610 \\
\hline Grmsnes-og Grafningshreppur & 8719 \\
\hline Hrunamannahreppur & 8710 \\
\hline Hverageri & 8716 \\
\hline Mýrdalshreppur & 8508 \\
\hline Ölfus & 8717 \\
\hline Skaftárhreppur & 8509 \\
\hline Sveitarflagi rborg & 8200 \\
\hline Uten navn & 9999 \\
\hline Vestmannaeyjar & 8000 \\
\hline Vestmannaeyjar & 8000 \\
\hline Grindavk & 2300 \\
\hline Reykjanesbr & 2000 \\
\hline Sandgeri & 2503 \\
\hline Vatnsleysustrandarhreppur & 2506 \\
\hline Arneshreppur & 4901 \\
\hline Bæjarhreppur & 4908 \\
\hline Bolungarvík & 4100 \\
\hline Ísafjarðarbær & 4200 \\
\hline Kaldrananeshreppur & 4902 \\
\hline Reykhólahreppur & 4502 \\
\hline Súðavíkurhreppur & 4803 \\
\hline Vesturbyggð & 4607 \\
\hline Akranes & 3000 \\
\hline Borgarfjararsveit & 3510 \\
\hline Dalabyggð & 3811 \\
\hline Eyja-og Miklaholtshreppur & 3713 \\
\hline Eyrarsveit & 3709 \\
\hline Helgafellssveit & 3710 \\
\hline Hvalfjararstrandarhreppur & 3501 \\
\hline Kjósarhreppur & 1606 \\
\hline Skorradalshreppur & 3506 \\
\hline Hvalfjarðarsveit & 3511 \\
\hline Borgarbyggð & 3609 \\
\hline Snæfellsbær & 3714 \\
\hline Hvtrsuhreppur & 3601 \\
\hline Innri-Akraneshreppur & 3503 \\
\hline Leirr-og Melahreppur & 3504 \\
\hline Saurbjarhreppur & 3809 \\
\hline Skilmannahreppur & 3502 \\
\hline Skorradalshreppur & 3506 \\
\hline Snfellsbr & 3714 \\
\hline rshafnarhreppur & 6709 \\
\hline Austur-Hra & 7620 \\
\hline Siglufjrur & 6250 \\
\hline Hörgárbyggð & 6514 \\
\hline
\end{tabular}


Broddaneshreppur

4911

Kolbeinsstaahreppur

3609

Grímsnes-og Grafningshreppur

8719

Laugardalshreppur

8721

Djprhreppur

8722

Austur-Eyjafjallahreppur

8613

Skeiða- og Gnúpverjahreppur

8720

Holta-og Landasveit

8614

Skagahreppur

5611 
2) Table S2. Table of vulnerable population for municipalities

\begin{tabular}{|c|c|c|}
\hline Name & Population & \% Exposed \\
\hline Reykjavík & 118814 & 23 \\
\hline Kópavogur & 31205 & 26 \\
\hline Seltjarnarnes & 4313 & 22 \\
\hline Garðabær & 13702 & 25 \\
\hline Hafnarfjörður & 26486 & 24 \\
\hline Mosfellsbær & 8854 & 23 \\
\hline Kjósarhreppur & 220 & 14 \\
\hline Reykjanesbær & 14137 & 24 \\
\hline Grindavíkurbær & 2830 & 13 \\
\hline Sandgerði & 1672 & 23 \\
\hline Sveitarfélagið Garður & 1477 & 23 \\
\hline Sveitarfélagið Vogar & 1126 & 26 \\
\hline Akranes & 6592 & 26 \\
\hline Skorradalshreppur & 60 & 30 \\
\hline Hvalfjarðarsveit & 627 & 20 \\
\hline Borgarbyggð & 3740 & 23 \\
\hline Grundarfjarðarbær & 899 & 21 \\
\hline Helgafellssveit & 57 & 39 \\
\hline Stykkishólmur & 1108 & 26 \\
\hline Eyja- og Miklaholtshreppur & 132 & 20 \\
\hline Snæfellsbær & 1737 & 23 \\
\hline Dalabyggð & 686 & 28 \\
\hline Bolungarvík & 889 & 25 \\
\hline Ísafjarðarbær & 3755 & 24 \\
\hline Reykhólahreppur & 271 & 30 \\
\hline Tálknafjarðarhreppur & 276 & 29 \\
\hline Vesturbyggð & 910 & 23 \\
\hline Súðavíkurhreppur & 182 & 23 \\
\hline Árneshreppur & 52 & 21 \\
\hline Kaldrananeshreppur & 104 & 18 \\
\hline Strandabyggð & 516 & 28 \\
\hline Sveitarfélagið Skagafjörður & 4024 & 24 \\
\hline Húnaping vestra & 1187 & 25 \\
\hline Blönduóssbær & 871 & 29 \\
\hline Sveitarfélagið Skagaströnd & 504 & 23 \\
\hline Skagabyggð & 104 & 28 \\
\hline Húnavatnshreppur & 412 & 21 \\
\hline Akrahreppur & 197 & 26 \\
\hline Akureyri & 17875 & 25 \\
\hline Norðurping & 2884 & 25 \\
\hline Fjallabyggð & 2035 & 26 \\
\hline Dalvíkurbyggð & 1900 & 25 \\
\hline Eyjafjarðarsveit & 1031 & 23 \\
\hline Hörgársveit & 584 & 23 \\
\hline
\end{tabular}




$\begin{array}{ccc}\text { Svalbarðsstrandarhreppur } & 390 & 22 \\ \text { Grýtubakkahreppur } & 350 & 26 \\ \text { Skútustaðahreppur } & 385 & 21 \\ \text { Tjörneshreppur } & 55 & 22 \\ \text { Pingeyjarsveit } & 915 & 27 \\ \text { Svalbarðshreppur } & 102 & 20 \\ \text { Langanesbyggð } & 512 & 28 \\ \text { Seyðisfjörður } & 677 & 23 \\ \text { Fjarðabyggð } & 4600 & 22 \\ \text { Vopnafjarðarhreppur } & 670 & 27 \\ \text { Fljótsdalshreppur } & 78 & 13 \\ \text { Borgarfjarðarhreppur } & 129 & 19 \\ \text { Breiðdalshreppur } & 190 & 23 \\ \text { Djúpavogshreppur } & 461 & 25 \\ \text { Fljótsdalshérað } & 3408 & 25 \\ \text { Sveitarfélagið Hornafjörður } & 2143 & 22 \\ \text { Vestmannaeyjar } & 4194 & 22 \\ \text { Sveitarfélagið Árborg } & 7783 & 26 \\ \text { Mýrdalshreppur } & 459 & 27 \\ \text { Skaftárhreppur } & 443 & 25 \\ \text { Ásahreppur } & 204 & 22 \\ \text { Rangárping eystra } & 1741 & 25 \\ \text { Rangárping ytra } & 1504 & 23 \\ \text { Hrunamannahreppur } & 774 & 24 \\ \text { Hveragerði } & 2283 & 26 \\ \text { Sveitarfélagið Ölfus } & 1930 & 21 \\ \text { Grímsnes- og Grafningshreppur } & 416 & 18 \\ \text { Skeiða- og Gnúpverjahreppur } & 504 & 22 \\ \text { Bláskógabyggð } & 906 & 22 \\ \text { Flóahreppur } & 602 & 22\end{array}$


3) Fig. S1. Thematic maps of milk (a) and wool (b) production and percentage of agricultural area (c) for municipalities
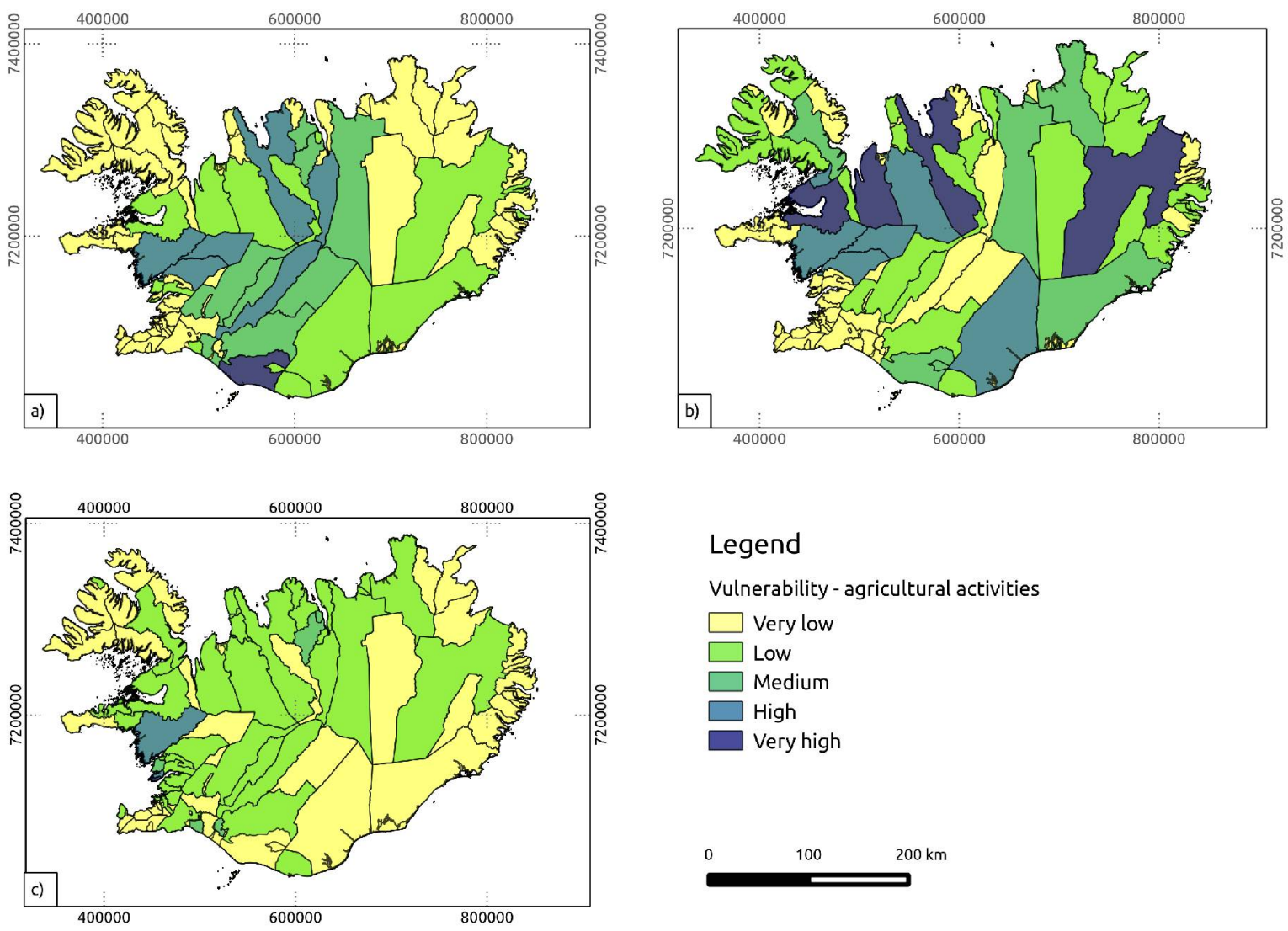

\section{Legend}

Vulnerability - agricultural activities

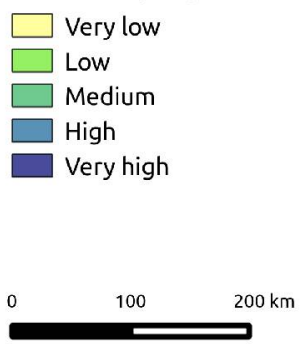


4) Table S3. List of airports considered for the vulnerability assessment of European airspace

\begin{tabular}{|c|c|c|c|c|c|c|}
\hline ICAO CODE & NAME & LON & LAT & YEAR & $\begin{array}{l}\text { FREIGHT } \\
\text { (tons) }\end{array}$ & PAX (number) \\
\hline EHAM & $\begin{array}{l}\text { AMSTERDAM } \\
\text { SCHIPHOL }\end{array}$ & 4.7642 & 52.3081 & 2012 & $1,510,925$ & $51,107,756$ \\
\hline EIDW & DUBLIN & -6.2700 & 53.4214 & 2012 & 111,070 & $19,090,781$ \\
\hline EGPH & EDINBURGH & -3.3725 & 55.9500 & 2012 & 42,938 & $9,195,305$ \\
\hline EDFH & $\begin{array}{c}\text { FRANKFURT } \\
\text { HAHN }\end{array}$ & 7.2642 & 49.9483 & 2012 & 239,297 & $2,926,419$ \\
\hline EGPF & GLASGOW & -4.4331 & 55.8719 & 2012 & 9,567 & $7,165,620$ \\
\hline EDDH & $\begin{array}{c}\text { HAMBURG } \\
\text { FUHLSBUETTEL }\end{array}$ & 9.9911 & 53.6303 & 2012 & 28,175 & $13,715,814$ \\
\hline EFHK & $\begin{array}{l}\text { HELSINKI- } \\
\text { VANTAA }\end{array}$ & 24.9633 & 60.3172 & 2012 & 188,549 & $14,872,568$ \\
\hline $\mathrm{BIKF}$ & KEFLAVIK & -22.6056 & 63.9850 & 2012 & 40,030 & $2,758,847$ \\
\hline EDDK & KOELN BONN & 7.1428 & 50.8658 & 2012 & 772,552 & $9,300,160$ \\
\hline EGKK & $\begin{array}{l}\text { LONDON } \\
\text { GATWICK }\end{array}$ & -0.1903 & 51.1472 & 2012 & 101,096 & $34,247,481$ \\
\hline EGLL & $\begin{array}{c}\text { LONDON } \\
\text { HEATHROW }\end{array}$ & -0.4614 & 51.4775 & 2012 & $1,556,219$ & $70,108,071$ \\
\hline EGGW & LONDON LUTON & -0.3683 & 51.8747 & 2012 & 29,636 & $9,621,081$ \\
\hline EGSS & $\begin{array}{c}\text { LONDON } \\
\text { STANSTED }\end{array}$ & 0.2350 & 51.8850 & 2012 & 237,783 & $17,498,891$ \\
\hline EGCC & MANCHESTER & -2.2750 & 53.3539 & 2012 & 98,270 & $19,819,851$ \\
\hline EGNX & $\begin{array}{l}\text { NOTTINGHAM } \\
\text { EAST MIDLANDS }\end{array}$ & -1.0792 & 52.9200 & 2012 & 299,994 & $4,083,919$ \\
\hline $\mathrm{EKCH}$ & $\begin{array}{l}\text { COPENHAGEN } \\
\text { KASTRUP }\end{array}$ & 12.6561 & 55.6181 & 2012 & 156,938 & $23,310,622$ \\
\hline ENGM & $\begin{array}{c}\text { OSLO } \\
\text { GARDERMOEN }\end{array}$ & 11.0839 & 60.2028 & 2012 & 76,326 & $22,196,044$ \\
\hline LFPG & $\begin{array}{l}\text { PARIS CHARLES } \\
\text { DE GAULLE }\end{array}$ & 2.5478 & 49.0097 & 2012 & $1,510,212$ & $61,648,325$ \\
\hline LFPO & PARIS ORLY & 2.3794 & 48.7233 & 2012 & 62,830 & $27,232,130$ \\
\hline ESSA & $\begin{array}{l}\text { STOCKHOLM } \\
\text { ARLANDA }\end{array}$ & 17.9186 & 59.6519 & 2012 & 74,219 & $19,721,791$ \\
\hline
\end{tabular}


5) Fig. S2. Thematic maps of air traffic and multi-modal accessibility values for European Nuts-2 regions: passengers (a) and freight (b), population (c) and multi-modal accessibility (d).
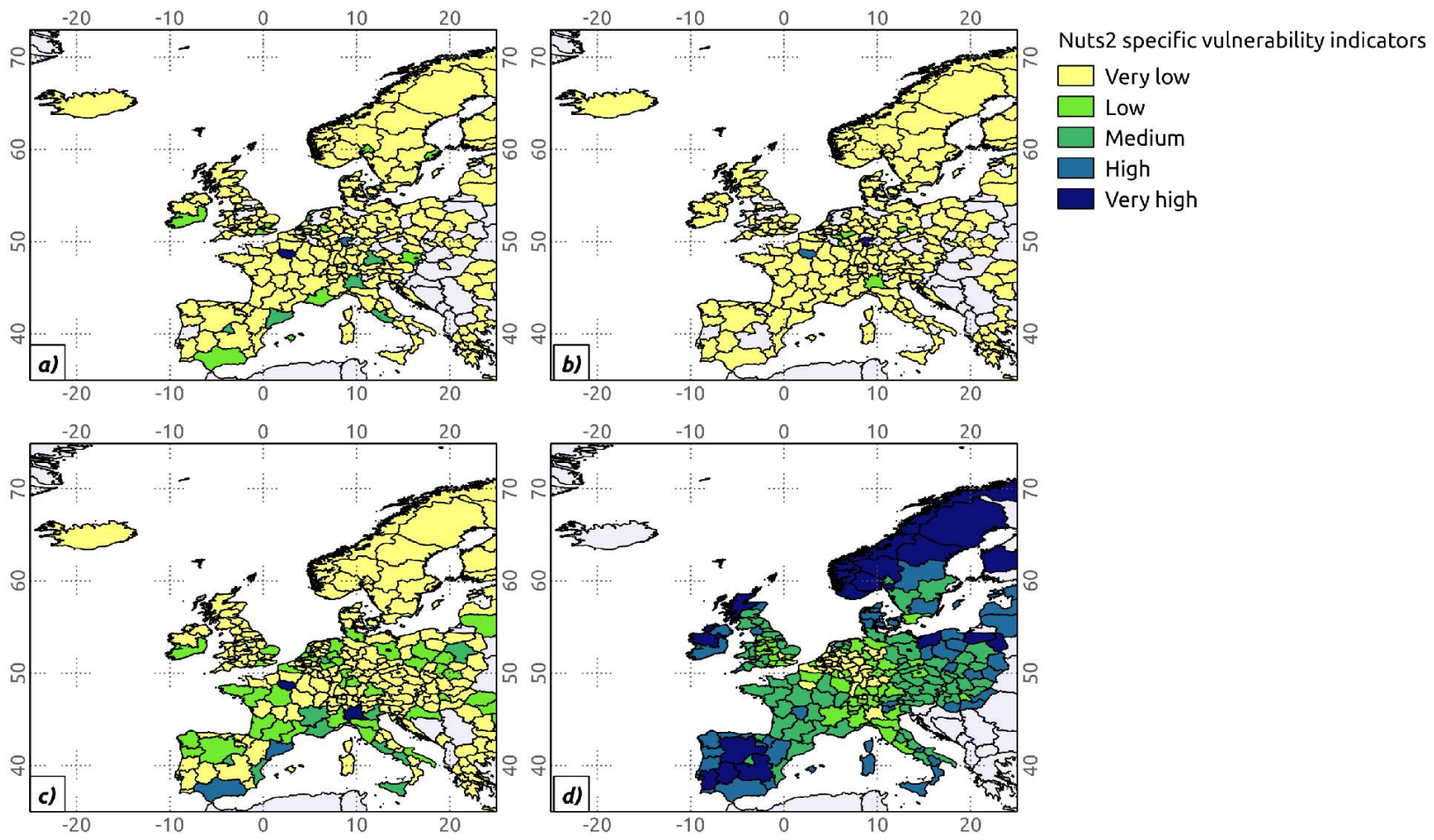
6) Fig. S3. Impact maps for FIRS produced at FL150 (top) and 300 (bottom) in case of occurrence of the considered eruptive scenarios: Hekla-2000 (a), Hekla-1947 (b), Katla-1918 (c) and Askja-1875 (d).
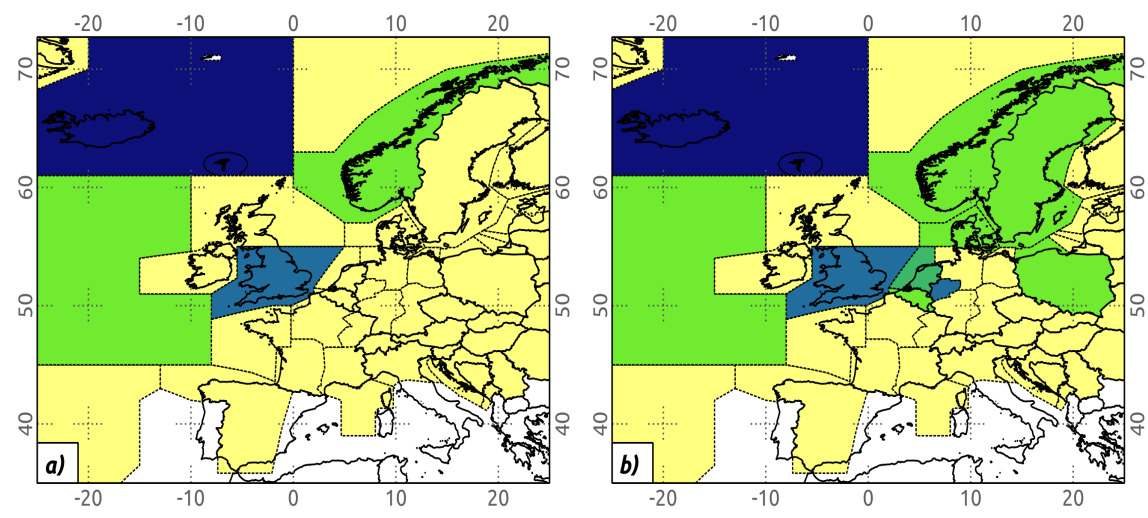

Impacts - FL150
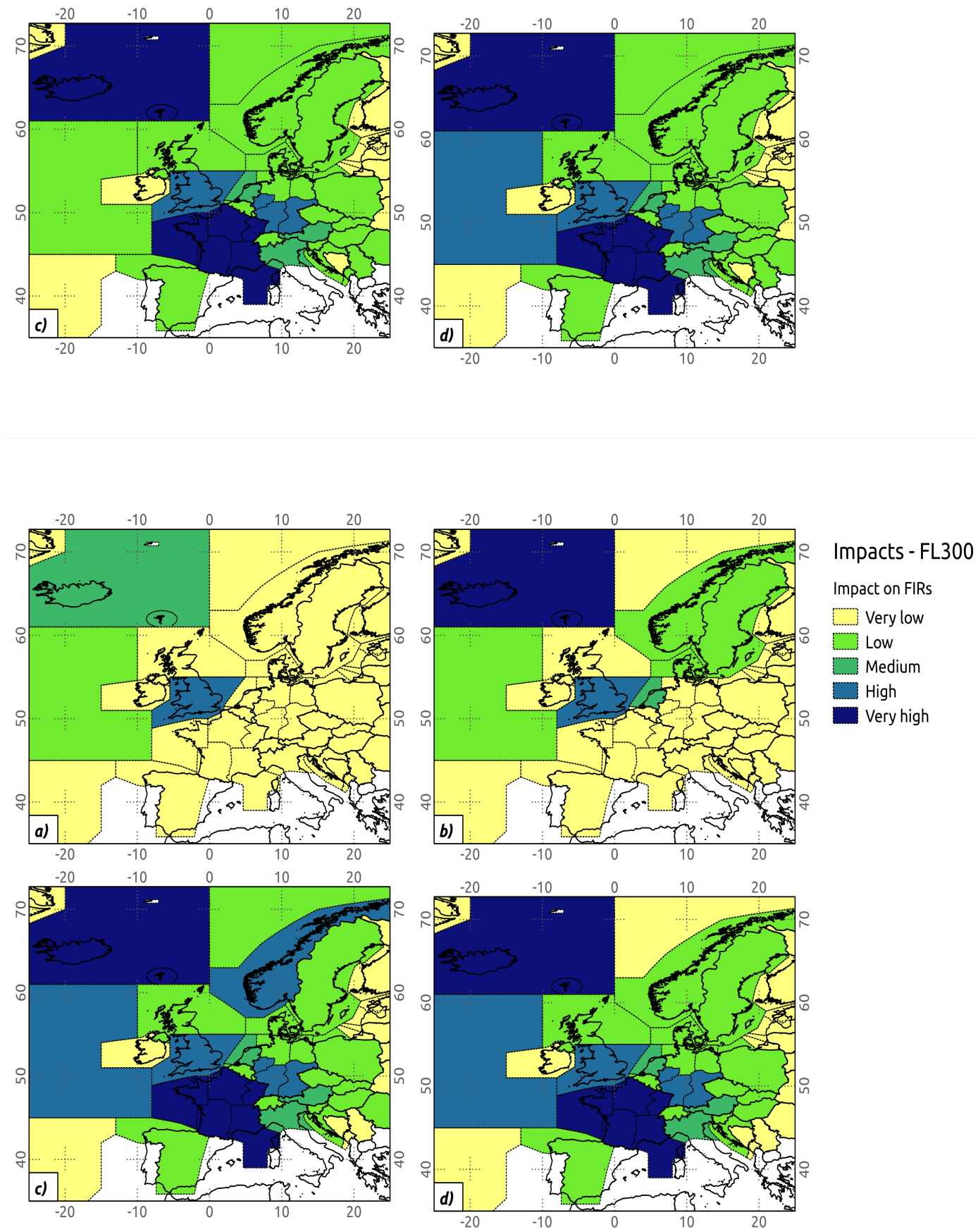